\title{
Evolution of magnetic polarons and spin-carrier interactions through the metal-insulator transition in $\mathrm{Eu}_{1-x} \mathrm{Gd}_{x} \mathrm{O}$
}

\author{
H. Rho, ${ }^{1}$ C. S. Snow, ${ }^{1}$ S. L. Cooper, ${ }^{1}$ Z. Fisk, ${ }^{2}$ A. Comment, ${ }^{1,3}$ and J-Ph Ansermet ${ }^{1,3}$ \\ ${ }^{1}$ Department of Physics and Frederick Seitz Materials Research Laboratory, \\ University of Illinois at Urbana-Champaign, Urbana, Illinois 61801 \\ ${ }^{2}$ National High Magnetic Field Laboratory, Florida State University, \\ 1800 East Paul Dirac Drive, Tallahassee, Florida 32306 \\ ${ }^{3}$ Ecole Polytechnique Fédérale de Lausanne, CH-1015 Lausanne, Switzerland
}

(Dated: November 4, 2018)

\begin{abstract}
Raman scattering studies as functions of temperature, magnetic field, and Gd-substitution are used to investigate the evolution of magnetic polarons and spin-carrier interactions through the metal-insulator transition in $\mathrm{Eu}_{1-x} \mathrm{Gd}_{x} \mathrm{O}$. These studies reveal a greater richness of phase behavior than have been previously observed using transport measurements: a spin-fluctuation-dominated paramagnetic $(\mathrm{PM})$ phase regime for $\mathrm{T}>\mathrm{T}^{*}>\mathrm{T}_{C}$, a two-phase regime for $\mathrm{T}<\mathrm{T}^{*}$ in which magnetic polarons develop and coexist with a remnant of the PM phase, and an inhomogeneous ferromagnetic phase regime for $\mathrm{T}<\mathrm{T}_{C}$.

PACS numbers: 78.30.-j, 71.30.+h, 75.
\end{abstract}

The remarkable phenomenon of "colossal magnetoresistance" (CMR) in perovskite-based oxides such as $\mathrm{La}_{1-x} \mathrm{~A}_{x} \mathrm{MnO}_{3}, \quad \mathrm{La}_{2-2 x} \mathrm{~A}_{1+2 x} \mathrm{Mn}_{2} \mathrm{O}_{7}$, and $\mathrm{La}_{1-x} \mathrm{~A}_{x} \mathrm{CoO}_{3}(\mathrm{~A}=\mathrm{Sr}, \mathrm{Ca})$ has renewed interest in the general subject of carrier-spin interaction effects in a much broader class of magnetic semiconductors, including ferromagnets such as $\mathrm{EuO}, \mathrm{EuS}$, and $\mathrm{EuB}_{6}$, and antiferromagnets like EuTe and EuSe [1]. In addition to large negative magnetoresistivities near $\mathrm{T}_{C}$, these Eubased systems share many intriguing properties with the higher $\mathrm{T}_{C}$ perovskite systems: a metal-insulator (MI) transition, and an accompanying ferromagneticparamagnetic (FM-PM) phase change, near $\mathrm{T}_{C}$; electronic phase-separation tendencies [2, 3]; magnetic-field induced transitions; etc. Consequently, these binary systems are particularly simple and well-controlled "laboratories" in which to explore carrier-spin interaction effects and FM cluster ("magnetic polaron") formation, as well as the impact of these phenomena on complex magnetic and electronic phase changes, in a broad class of magnetic systems [任].

The $\mathrm{Eu}_{1-x} \mathrm{Gd}_{x} \mathrm{O}$ system is particularly interesting to study for several reasons. Conductivity and magnetic susceptibility measurements have shown that Eu-rich $\mathrm{EuO}$ exhibits a FM-PM phase change below $\mathrm{T}_{C} \sim 69 \mathrm{~K}$ [5, 6], affording an opportunity to investigate spectroscopically the nature of transitions between various complex phases as functions of temperature and magnetic field. Furthermore, substitution of Gd provides a means of elevating both $\mathrm{T}_{C}$ [7] and the conductivity [5] in a systematic and controlled manner, enabling one to study the effects of both $\mathrm{T}_{C}$ and disorder on spin-carrier interactions and magnetic polaron formation.

In this Letter, we present an inelastic light (Raman) scattering study of spin-carrier interactions and magnetic polaron formation in the $\mathrm{Eu}_{1-x} \mathrm{Gd}_{x} \mathrm{O}$ system as functions of temperature, magnetic field, and Gd substitution. Raman scattering is a particularly effective technique for studying spin-carrier interactions in magnetic systems, as it affords a unique means of simultaneously investigating both the carrier dynamics and spin excitations in various phases of these materials 8, 9. More particularly, spinflip (SF) Raman scattering provides a sensitive and direct means by which magnetic polarons can be detected and studied in different phases of magnetic semiconductors [8, 9, 10, 11, 12, 13]. For example, the Raman scattering study presented here demonstrates that the phase behavior in $\mathrm{Eu}_{1-x} \mathrm{Gd}_{x} \mathrm{O}$ is much richer near $\mathrm{T}_{C}$ than has been previously evident from transport measurements: it consists of a high-temperature PM regime in which electronic scattering is dominated by spin fluctuations, a "cluster formation" temperature regime $\left(\mathrm{T}<\mathrm{T}^{*}\right)$ in which magnetic polarons develop and coexist with PM regions, and finally an inhomogeneous FM metal regime $\left(\mathrm{T}<\mathrm{T}_{C}\right)$. The results of this study further suggest that both the stability and exchange energy associated with the magnetic polarons are enhanced by Gd-substitution and by the application of a magnetic field.

Raman scattering measurements were performed with $\quad \mathrm{Eu}_{1-x} \mathrm{Gd}_{x} \mathrm{O}$ samples $\quad\left(\mathrm{x}=0.006: \mathrm{T}_{C} \sim 70 \mathrm{~K}\right.$; $\left.\mathrm{x}=0.035: \mathrm{T}_{C} \sim 115 \mathrm{~K}\right)$ mounted inside a variabletemperature, continuous helium-flow cryostat, which allowed Raman studies at temperatures ranging from $4 \mathrm{~K}$ to $350 \mathrm{~K}$, and in magnetic fields up to $8 \mathrm{~T}$. Samples were excited in a true-backscattering geometry using the $647.1 \mathrm{~nm}$ excitation wavelength of a $\mathrm{Kr}$-ion laser. For $\mathrm{H}=0$ measurements, linearly polarized light was employed in $z(x x) \bar{z}$ and $z(x y) \bar{z}$ configurations, where $x\|[1,0,0], y\|[0,1,0]$, and $z \|[0,0,1]$. In the notation $z(x y) \bar{z}, z$ and $\bar{z}$ represent the wavevector directions of the incident and scattered light, respectively, and $(x, y)$ represents the polarization directions of the incident 


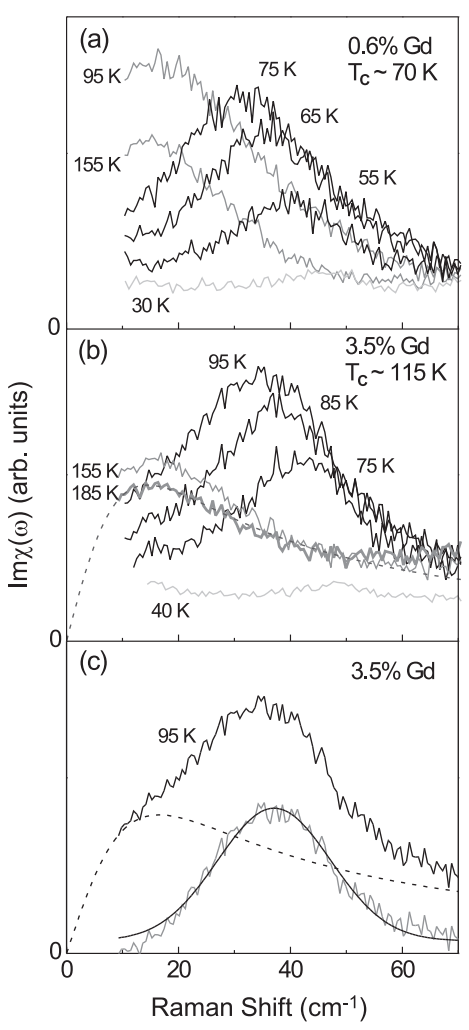

FIG. 1: Raman scattering spectra as a function of temperature for $\mathrm{Eu}_{1-x} \mathrm{Gd}_{x} \mathrm{O}$ samples with (a) $\mathrm{x}=0.006$ and (b) $\mathrm{x}=0.035$. A dashed line in (b) indicates a fitting result using Eq. 1 for the Raman spectrum at $185 \mathrm{~K}$. The extended portion of the fitting curve toward $0 \mathrm{~cm}^{-1}$ is a guide for the eye. (c) SF Raman response (bottom gray curve), which has been fit with a Gaussian profile (dark solid line), after removing collision-dominated contribution (dashed line) from the raw $\mathrm{T}=95 \mathrm{~K}$ spectrum (top curve).

and scattered light, respectively. For magnetic field dependent measurements, circularly polarized light was employed in a $z(L R) \bar{z}$ configuration, where $L$ and $R$ represent left and right circularly polarized light, respectively. The crystallographic axes of each sample were identified by Laue x-ray diffraction, and the Curie temperatures of the samples were determined with magnetic susceptibility measurements using SQUID-based magnetometers.

Conductivity measurements of $\mathrm{EuO}$ [5, 6] can distinguish two distinct temperature regimes in this system: a regime above $\mathrm{T}_{C}(\sim 69 \mathrm{~K})$ in which the resistivity exhibits activated behavior and a large negative magnetoresistivity, and a regime below $\mathrm{T}_{C}$ in which the conductivity increases by roughly 13 orders-of-magnitude as the system transitions into the FM metal phase. By contrast, as shown in Figs. 1(a) and (b), Raman scattering measurements are able to resolve additional richness in the phase behavior of this system, revealing distinct spectroscopic signatures associated with three different phase regimes as a function of temperature: (i) a high temperature "spin-fluctuation-dominated" PM regime for $\mathrm{T}_{C}<\mathrm{T}<\mathrm{T}_{s f}$, where $\mathrm{T}_{s f}$ is the temperature below which carrier scattering is dominated by spin fluctuations, (ii) a "magnetic polaron" regime in which magnetic polarons develop below a formation temperature $\mathrm{T}^{*}$ $\left(\mathrm{T}_{C}<\mathrm{T}^{*}<\mathrm{T}_{s f}\right)$, and (iii) an inhomogeneous $\mathrm{FM}$ regime for $\mathrm{T}<\mathrm{T}_{C}$ in which a $\mathrm{FM}$ metal component coexists with a diminishing density of magnetic polarons. Below, we consider each of these phase regimes, and the transitions between them, in greater detail.

The high temperature PM phase is characterized in the Raman spectrum by a collision-dominated electronic scattering response [8, 9, 14],

$$
S(\omega) \propto(1+n(\omega)) \operatorname{Im} \chi(\omega)=(1+n(\omega)) \frac{\left|\gamma_{L}\right|^{2} \omega \Gamma_{L}}{\omega^{2}+\Gamma_{L}^{2}},
$$

where $1+n(\omega)$ is the Bose thermal factor, $L$ is the scattering channel selected by a particular scattering geometry, $\gamma_{L}$ is the Raman scattering vertex in channel $\mathrm{L}$, and $\Gamma_{L}$ is the electronic scattering rate in channel L. Fig. 1 (b) illustrates that the collision-dominated response in Eq. 1 indeed provides an excellent fit (dashed line) to the Raman spectra in the PM phase. In conventional semiconductors, the scattering rate is typically associated with impurity scattering due to extrinsic defects or vacancies 15. However, in magnetic semiconductors such as $\mathrm{EuO}$ and $\mathrm{EuB}_{6}$, for temperatures sufficiently close to $\mathrm{T}_{C}$, electronic scattering is dominated by short-range spin fluctuations. Consequently, we find in this regime that the scattering rate scales according to $\Gamma \sim \chi(T) T$ [9], where $\chi(T)$ is the temperature-dependent magnetic susceptibility, and we also find that the intensity of the collision-dominated scattering response increases substantially with decreasing temperature towards $\mathrm{T}_{C}$. Significantly, the resistivity of $\mathrm{Eu}_{1-x} \mathrm{Gd}_{x} \mathrm{O}$ [16] exhibits activated behavior between room temperature and $\mathrm{T}_{C}$, which also suggests the dominance of "critical" electronic scattering from spin fluctuations throughout this temperature regime. However, the Raman results in Fig. 1 (a) and (b) reveal additional complexity in the $\mathrm{H}=0$ phase behavior of $\operatorname{Eu}_{1-x} \operatorname{Gd}_{x} \mathrm{O}$ above $\mathrm{T}_{C}$ : below a temperature $\mathrm{T}^{*}\left(>\mathrm{T}_{C}\right)$, there is a striking change in the Raman response, from a collision-dominated low frequency response to an inelastic response with a clear Gaussian profile. Significantly, this inelastic response develops in the $(x, y)$ and $(x+y, x-y)$ [i.e., $\mathrm{E}_{i} \perp \mathrm{E}_{s}$ ] scattering geometries, but not in the $(x, x)$ [i.e., $\mathrm{E}_{i} \| \mathrm{E}_{s}$ ] scattering geometry, and hence has the transformation properties of the totally antisymmetric Raman tensor. Numerous previous investigations in both dilute and dense magnetic semiconductors have identified this distinctive response as $\mathrm{H}=0$ SF Raman scattering associated with the development of magnetic polarons [8, 9, 10, 11, 12, 13. Hence, the development of this Raman response in the $\mathrm{Eu}_{1-x} \mathrm{Gd}_{x} \mathrm{O}$ system betrays a distinct regime above $\mathrm{T}_{C}$ in which local FM clusters nucleate prior to the development of the FM ground state. As a function of decreasing temperature below $\mathrm{T}_{C}$, the $\mathrm{H}=0 \mathrm{SF}$ Raman 

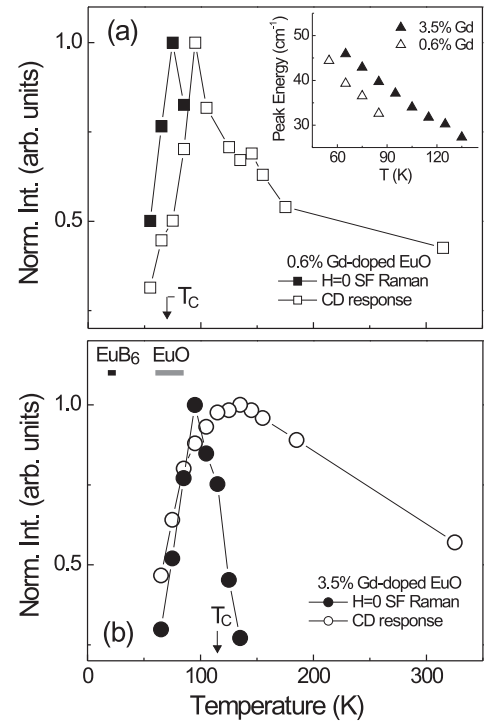

FIG. 2: Spectrally integrated intensity changes as a function of temperature both for collision-dominated scattering response and for magnetic polaron response in $\mathrm{Eu}_{1-x} \mathrm{Gd}_{x} \mathrm{O}$ with (a) $\mathrm{x}=0.006$ and (b) $\mathrm{x}=0.035$. Horizontal bars in (b) denote temperature ranges in which magnetic polarons form in $\mathrm{EuO}$ and $\mathrm{EuB}_{6}$ for a comparison purpose. The inset in (a) shows polaron peak energy changes as a function of temperature for $\mathrm{x}=0.006$ and 0.035 .

response gradually decreases in intensity as the system transitions into the FM metal phase, reflecting the gradual dissolution of localized magnetic polarons with increasing spin order in the FM phase as the localization length for the magnetic polarons diverges. Below $50 \mathrm{~K}$, the Raman intensity associated with magnetic polarons decreases rapidly due to the saturation of spins.

Figures 1 and 2 reveal several important features of magnetic polaron evolution in $\mathrm{Eu}_{1-x} \mathrm{Gd}_{x} \mathrm{O}$. First, the $\mathrm{H}=0$ SF Raman energy increases systematically with decreasing temperature, indicative of magnetic polarons in a spin-aligned "cooperative" regime, in which the spins of the carriers and magnetic ions are cooperatively aligned in the FM clusters 10, 11, 17. Second, careful fits to the spectra, illustrated in Fig. 1 (c) and summarized in Fig. 2, demonstrate that the magnetic polaron regime is characterized by the coexistence of $\mathrm{H}=0 \mathrm{SF}$ (inelastic Gaussian) and collision-dominated electronic (Eq.1) scattering responses, providing strong evidence that this is a "two-phase" regime in which FM clusters coexist with some remnant of the PM phase. This behavior is summarized for both $\mathrm{x}=0.006$ and $\mathrm{x}=0.035$ samples in Fig. 2, which compares the integrated intensities of both the $\mathrm{H}=0 \mathrm{SF}$ (filled symbols) and collision-dominated scattering (open symbols) responses as a function of temperature. Finally, note that the polarons in $\mathrm{Eu}_{1-x} \mathrm{Gd}_{x} \mathrm{O}$ are stable over a temperature range that is $\sim 5-10$ times higher than in $\mathrm{EuB}_{6}$ 88, 9]. Furthermore, both $T^{*}$ and the temperature range over which polarons are stable increase with increasing Gd-concentration. These results provide direct evidence that increased spin-disorder sta-
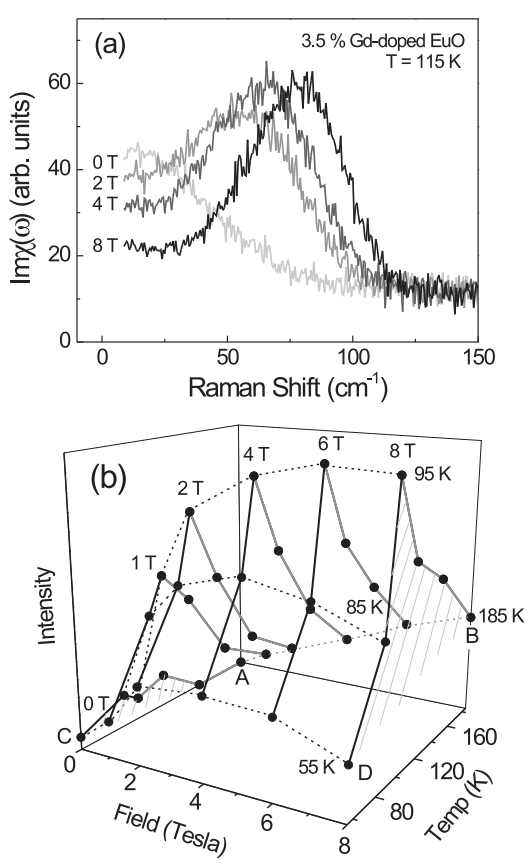

FIG. 3: (a) Magnetic field dependent Raman scattering at $115 \mathrm{~K}$. Note that in a $z(L R) \bar{z}$ scattering configuration, the collision-dominated scattering response prevails in spectra when $\mathrm{H}<1 \mathrm{~T}$, that makes unable to identify more detail on the SF Raman response. (b) Spectrally integrated intensity profile of the SF Raman response as functions of magnetic field and temperature. Data points observed at the same field (temperature) are connected by the solid (dotted) lines.

bilizes magnetic polarons in the CMR-type systems.

It is of great interest to examine the effects of an externally applied field on the low frequency excitation spectra near $\mathrm{T}_{C}$ in Eu-based compounds, and more particularly on the magnetic polarons in these systems. The field dependence of the Raman spectrum is illustrated for $\mathrm{T}=115 \mathrm{~K}$ in Fig. 3 (a), which shows the evolution of a SF Raman response with increasing magnetic field. The integrated intensity of the SF Raman response is summarized as functions of both the temperature and magnetic field in Fig. 3(b). Figures 3 (a) and (b) show two particularly interesting effects of the magnetic field on the SF Raman spectrum in $\mathrm{Eu}_{0.965} \mathrm{Gd}_{0.035} \mathrm{O}$. First, both the integrated intensity and the energy of the SF Raman response increase with increasing field, suggesting that there is a corresponding increase in the effective d-f exchange energy within the magnetic cluster. Second, the collision-dominated electronic response diminishes with increasing field, reflecting the decrease of spinfluctuations at high fields. The SF Raman intensity "surface" shown in Fig. 3 (b) also reveals several features: (a) in the PM phase, increasing the magnetic field from $\mathrm{H}=0$ (i.e., from point $\mathrm{A}$ to $\mathrm{B}$ at $\mathrm{T}=185 \mathrm{~K}$ ) results in the development and linear increase in the SF Raman intensity, suggesting that a magnetic field stabilizes the formation of magnetic polarons at high temperature, presumably by both increasing the magnetic susceptibility at these 


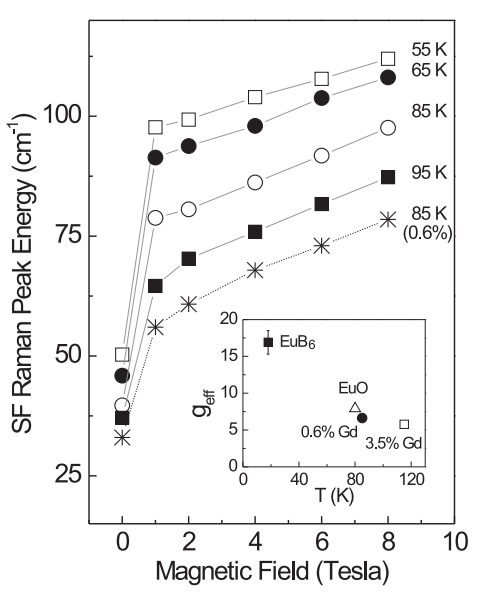

FIG. 4: SF Raman peak energy changes as a function of applied magnetic field at various temperatures for $\mathrm{Eu}_{1-x} \mathrm{Gd}_{x} \mathrm{O}$ with $\mathrm{x}=0.006$ (stars) 0.035 (squares and circles). The inset shows effective g-value obtained from linear fits in the field range of $1 \leq \mathrm{H} \leq 8 \mathrm{~T}$.

temperatures and reducing thermal fluctuations; and (b) increasing the magnetic field from $\mathrm{H}=0$ well below $\mathrm{T}_{C}$ (e.g., from point $\mathrm{C}$ to $\mathrm{D}$ at $\mathrm{T}=55 \mathrm{~K}$ ) causes the $\mathrm{SF}$ Raman intensity to increase initially at lower fields, then decrease for higher fields, suggesting that the system is close to the FM phase regime in which the polarons become ionized at these high fields and low temperatures. This trend is similar to that observed in $\mathrm{EuB}_{6}$ at high fields [8, 9].

In order to examine more quantitatively the effects of a magnetic field on the magnetic polarons in $\mathrm{Eu}_{1-x} \mathrm{Gd}_{x} \mathrm{O}$, we plot in Fig. 4 the SF Raman energy as a function of field at several temperatures, and for both $0.6 \%$ Gd(stars) and 3.5\% Gd- (squares and circles) substituted $\mathrm{EuO}$. To simplify the interpretation of these data, we consider only field-dependent data at those temperatures for which there is an $\mathrm{H}=0 \mathrm{SF}$ Raman response, i.e., for which there are magnetic polarons present even at $\mathrm{H}=0$. The summary in Fig. 4 illustrates several interesting points. First, there is an abrupt jump in the SF Raman peak energy between $0-1$ Tesla, followed by a linear increase in the SF energy with increasing magnetic field. As the SF Raman energy is given by $\hbar \omega_{0} \propto J_{d f}\langle M\rangle$, the increase with increasing field reflects an enhancement in the exchange energy associated with the polarons due to increased polarization of the spins. The origin of the abrupt jump with small applied field is not clear, but it is worth noting that the individual moments of the spontaneously-formed magnetic polarons are randomly oriented at $\mathrm{H}=0$, but are expected to become rapidly aligned upon application of a field 佃. Hence, the abrupt jump in the SF Raman energy appears to reflect an enhancement in the effective field experienced by localized charges when the magnetic polarons become mutually-aligned with the application of a field. Second, Fig. 4 illustrates that the SF Raman energy increases both with decreasing temperature and with increasing
Gd concentration, again presumably reflecting the larger effective magnetization associated with the polarons (i.e., larger polaron sizes) under these conditions [1, 18]. Using the slope obtained from the linear-in-field "high-field" regime $(1 \leq \mathrm{H} \leq 8 \mathrm{~T})$, we can estimate an effective g-value $\left(g_{\text {eff }} \sim \Delta \mathrm{E} / \mu_{B} \Delta \mathrm{H}\right)$ of $\sim 6$ for $0.6 \%$ and $3.5 \%$ (Eu,Gd)O at $\mathrm{T} \sim \mathrm{T}_{C}$. By contrast, the much less disordered system $\mathrm{EuB}_{6}$ has $\mathrm{g}_{\text {eff }} \sim 17$ for $\mathrm{T} \sim \mathrm{T}_{C}$, suggesting that the polaron size in $(\mathrm{Eu}, \mathrm{Gd}) \mathrm{O}$ may be fundamentally much more limited by intrinsic spin disorder than in $\mathrm{EuB}_{6}$. Indeed, the PM semimetal to FM metal transition in $\mathrm{EuB}_{6}$ appears to occur via a continuous evolution - and eventual percolation - of the magnetic polarons [8, 9], whereas the polaronic SF Raman response in (Eu,Gd)O simply appears to dissipate into the FM phase (see Fig. 3(b)) with decreasing temperature.

In summary, Raman scattering studies reveal direct evidence for diverse phase behavior in $\mathrm{Eu}_{1-x} \mathrm{Gd}_{x} \mathrm{O}$, most notably a coexistence regime involving magnetic polarons and $\mathrm{PM}$ phase regions in the vicinity of $\mathrm{T}_{C}$. This regime is found to be stabilized both by increasing magnetic field and the substitution of magnetic impurities. These results demonstrate, most significantly, that electronic inhomogeneity and cluster formation are not unique to complex, perovskite-related oxides [4, but occur rather generally even in structurally simple (binary) systems that exhibit a similar strong competition between carrier kinetic and magnetic interaction energies.

We thank M. V. Klein for useful discussions. We acknowledge support of this work by the Department of Energy (DEFG02-96ER45439) and the National Science Foundation (DMR97-00716).

[1] For a review of these materials, see E. L. Nagaev, Physics Reports, 346, 387 (2001) and T. Kasuya and A. Yanase, Rev. Mod. Phys. 40, 684 (1968).

[2] E. L. Nagaev, Phys. Stat. Sol. B 186, 9 (1994).

[3] A. Moreo et al., Phys. Rev. Lett. 84, 5568 (2000); A. Moreo et al., Science 283, 2034 (1999).

[4] J. Burgy et al., arXiv:cond-mat/0107300 (2001).

[5] M. R. Oliver et al., Phys. Rev. B 5, 1078 (1972).

[6] J. B. Torrance et al., Phys. Rev. Lett. 29, 1168 (1972).

[7] A. Mauger et al., J. Phys. (Paris) 41, C5-263 (1980).

[8] P. Nyhus et al., Phys. Rev. B 56, 2717 (1997).

[9] C. S. Snow et al., to be published in Phys. Rev. B.

[10] E. D. Isaacs et al., Phys. Rev. B 37, 7108 (1988).

[11] D. Heiman et al., Phys. Rev. B 27, 4848 (1983).

[12] D. L. Peterson et al., Phys. Rev. B 32, 323 (1985).

[13] A. K. Ramdas and S. Rodriguez, in Light Scattering in Solids VI, edited by M. Cardona and G. Güntherodt (Springer-Verlag, Berlin, 1991), p. 137.

[14] A. Zawadowski and M. Cardona, Phys. Rev. B 42, 10732 (1990).

[15] M. V. Klein, in Light Scattering in Solids, edited by M. Cardona (Springer-Verlag, Berlin, 1975), p. 150.

[16] S. von Molnar and M. W. Shafer, J. Appl. Phys. 41, 1093 (1970).

[17] J. Warnock and P. A. Wolff, Phys. Rev. B 31, 6579 (1985).

[18] C. B. à la Guillaume, Physica 146B, 234 (1987). 